\title{
A Network Communication Protocols for Robotic-assisted Vascular Intervention Systems
}

\author{
Quan ZENG ${ }^{1}$, Shou-jun ZHOU ${ }^{2}$, Hao SHEN ${ }^{3}$ and Cheng WANG ${ }^{2, a,{ }^{*}}$ \\ ${ }^{1}$ College of Information Engineering, Shen Zhen University, China \\ ${ }^{2}$ Shenzhen Institutes of Advanced Technology, Chinese Academy of Sciences, China \\ ${ }^{3}$ Material Science and Engineering School, Shanghai Jiao Tong University, China \\ aquan.zeng@siat.ac.cn, ${ }^{b}$ sj.zhou@siat.ac.cn, ${ }^{\mathrm{c}}$ shellon@sjtu.edu.cn \\ acheng.wang@siat.ac.cn \\ ${ }^{*}$ Corresponding author
}

Keywords: MIS, Robot, Communication Network protocol, Real-time

\begin{abstract}
In recent years, minimally invasive surgery (MIS) has become a very suitable field for robotic systems. Minimally invasive surgery which can be used for diagnosis and surgical treatment is more and more popular in the medical field. We have developed a remote vascular intervention robot system for medical applications which is integrated by several modules. During the remote operation of intervention therapy, we need to transmit CT images, robot instructions, force feedback data to make the surgery smoothly. So, we have developed an open, simple and extensible peer-to-peer communication protocol. Experiments result show that the network communication protocol can meet the requirements of real-time capability in the surgery.
\end{abstract}

\section{Introduction}

In recent years, minimally invasive surgery (MIS) has become a very suitable field for robot systems [1]. Minimally invasive surgery in the medical field is more and more popular, which can be used for diagnosis and surgery. Many procedures of endoscopes or catheters, being used for diagnosis and medical surgery, are now possible in minimally invasive surgery [2]. Compared with open treatment, when in MIS treatment, patients suffer small incision, recover quickly, do not need general anesthesia, low risk; in addition, it can deliver the drug to the specified location, improve drug efficacy and reduce damage to other organizations [3]. However, the clinician who are exposed to radiation for a long time can't avoid the risk of cataract and cancer caused by radiation, even if wearing a lead protective clothes [3].

The robotic-assisted minimally invasive surgical robotic system is now able to perform multiple types of surgery, such as skull base surgery [4], mandibular reconstruction surgery [5], vascular surgery [6,7] and so on. And the current trend of medical robots is to develop telephoto medical robots. It describes a variety of remote operation medical robots in [8], such as the most prestigious Da Vinci robots. Teleoperation Medical robot consists of multiple devices that require the transfer of information between each other. In [9], a distributed modular architecture has been developed that can be applied to a variety of surgical robotic systems, providing a reference for telemedicine robots. And with the robot-assisted vascular intervention robot deep study, to develop a standard, open and expandable communication protocol is even more urgent. In [10] Junichi Tokuda et al have developed a set of standard communication protocols for sharing data between devices and software 
during image navigation. It is a standard, open and extensible communication protocols. This protocol meets the real-time requirements, whether there is a type or two types of data transmitted at the same time. The latency in message transmission is within the allowable range, meeting real-time requirements, and used in some cases. But it is only applied to the image navigation robot-assisted interventional surgical system, and did not establish a secure communication mechanism. In this paper a standard, simple and extensible peer-to-peer communication protocol will be proposed. It is suitable for any robot-assisted interventional surgical system, not just to meet the impact of navigation vascular intervention system, which is more universal.

\section{Method}

In order to meet the communication requirements of the master-slave teleoperation of the vascular interventional robot, this paper defines a set of standard communication protocols, including the establishment, management and closure of the communication. According to the communication protocol network architecture based on TCP / IP peer-to-peer network communication, the points communicate with each other by the socket asynchronously. peer-to-peer network communication can make the communication efficiency improved significantly. At the same time, this set of communication protocols is suitable in a variety of vascular interventional robot systems, such as image-guided vascular intervention surgery robot system and the vascular intervention surgery robot system by magnetic navigation.

\section{Description of the Message}

In this communication protocol, the minimum data transmission unit is defined as a message. There are many types of data packets, but basically composed of two parts, the header and the body of the message, as shown in Figure 1. As the vascular intervention surgery robot system consists of multiple devices, each device needs to communicate with each other. Different types of message are defined in this communication protocol. Regardless of the type of message, the size of the header of the message is a fixed number of bytes. It is mainly used to identify the source of the information transmission, to store the basic information of the source, including the data type, the identifier of the device, the timestamp and the length of the data. And the body of message is variable, according to the data type.

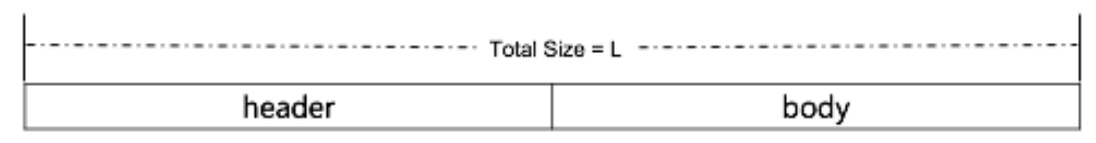

Figure 1. Composition of message

Header. The header of the message plays a key role in the process of information transmission, which contains the basic information of the message. When the receiver receives the data message, the receiver can correctly identify the message by relying on the header information of the message, and decode the message. The size of the header of the message is fixed, including the data type of the message, the identifier of the device that sends the message, the recipient identifier of the message, the timestamp, and the length of the message. The message header is shown in Figure 2. 


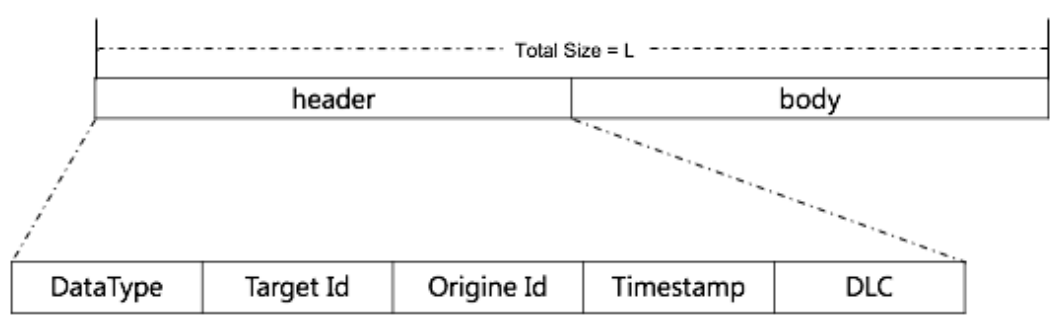

Figure 2. Composition of the message header

The basic role of the various parts of the message header is shown in Table 1

Table 1 the functions of the various parts of the message header

\begin{tabular}{|c|c|c|}
\hline Name & Data Type & Description \\
\hline Datatype & Byte [2] & $\begin{array}{l}\text { Two bytes ASCII code that represents the type of message. data } \\
\text { types are mainly divided into two types: session management } \\
\text { message and robotic information message. It contains } 2^{16} \text { types, } \\
\text { which is sufficient for future data type extensions. For example, } \\
\text { the following values will be determined: } \\
\text { HSD HCD Two message to establish a session. } \\
\text { HLD HelloMessage that diagnoses whether the } \\
\text { session is smooth. } \\
\text { CSD CloseSession Message }\end{array}$ \\
\hline OrigineID & Byte & A byte ASCII code identifies the sender of the message. \\
\hline TargetID & Byte & $\begin{array}{l}\text { A byte ASCII code identifies the receiver of the message and can } \\
\text { be used to verify whether the message is transmitted to the } \\
\text { specified device. }\end{array}$ \\
\hline Timestamp & UInt32 & $\begin{array}{l}\text { An unsigned integer represents the timestamp when the message } \\
\text { is generated, which can be used to calculate the latency of the } \\
\text { transmission. }\end{array}$ \\
\hline DLC & Byte [2] & $\begin{array}{l}\text { The length of the message, in byte, is represented by a two-byte } \\
\text { ASCII code, excluding the header of the data message. }\end{array}$ \\
\hline
\end{tabular}

Body. The body of the message will vary depending on the data type of the message. The data type of the message is divided into two types: 1. the data type manage the communication session; 2 . The data type obtains the robot information. The data types are classified as shown in Table 2. Because the size of Datatype is two bytes, containing 216 types message, which can satisfy the requirement in the communication process of the vascular intervention system. The number of message that manages communication session connected is not too many, therefore the range of $0 \sim 255$ is reserved. At the same time, 256 (216-1) is reserved to represent the second type of message about the robot information, so that the communication protocol can be used to provide sufficient room for expansion future. 
Table 2 Classification and function of message data types

\begin{tabular}{|c|c|c|c|}
\hline Class & Function & Name & Description \\
\hline \multirow{7}{*}{$\begin{array}{l}\text { Manage } \\
\text { session }\end{array}$} & \multirow{2}{*}{$\begin{array}{l}\text { Establish } \\
\text { Session }\end{array}$} & HandShakeMsg & Establish Session with each device \\
\hline & & HandShakeCommitMsg & Confirm establishment of session \\
\hline & \multirow{2}{*}{$\begin{array}{l}\text { Close } \\
\text { Session }\end{array}$} & CloseSessionMsg & Close session actively \\
\hline & & CloseSessionCommitMsg & Confirm that the session is closed \\
\hline & \multirow[t]{2}{*}{ Diagnosis } & HelloMsg & $\begin{array}{l}\text { Diagnose whether the session is } \\
\text { normal }\end{array}$ \\
\hline & & $\ldots$ & \\
\hline & \multicolumn{3}{|l|}{$\ldots$} \\
\hline \multirow{6}{*}{$\begin{array}{l}\text { Robot } \\
\text { system } \\
\text { message }\end{array}$} & \multirow{4}{*}{$\begin{array}{l}\text { Medical } \\
\text { Image }\end{array}$} & CT/DSA/MRI & Transmit medical image message \\
\hline & & .. & \\
\hline & & Progress/Rotate & $\begin{array}{l}\text { Transmit message that control robot } \\
\text { movement }\end{array}$ \\
\hline & & $\ldots$ & \\
\hline & \multicolumn{2}{|c|}{ System status } & Monitor system status \\
\hline & \multicolumn{2}{|l|}{ Position } & $\begin{array}{l}\text { Transfer the location of the NDI } \\
\text { needle which is fixed to the head of } \\
\text { the guide wire }\end{array}$ \\
\hline
\end{tabular}

Medical image: This message is used to transmit image data, such as CT, DSA and MRI. In the vascular intervention surgery robot system, video navigation can help us determine the movement of the guide wire. The movement of the guide wire can be controlled accurately, so that vascular intervention surgery will be operated more smoothly. The body of the medical image message will be divided into the image header and the image body, as shown in Figure 3. The image header represents some of the parameter information of the image, such as the length, width, and height of the medical image.

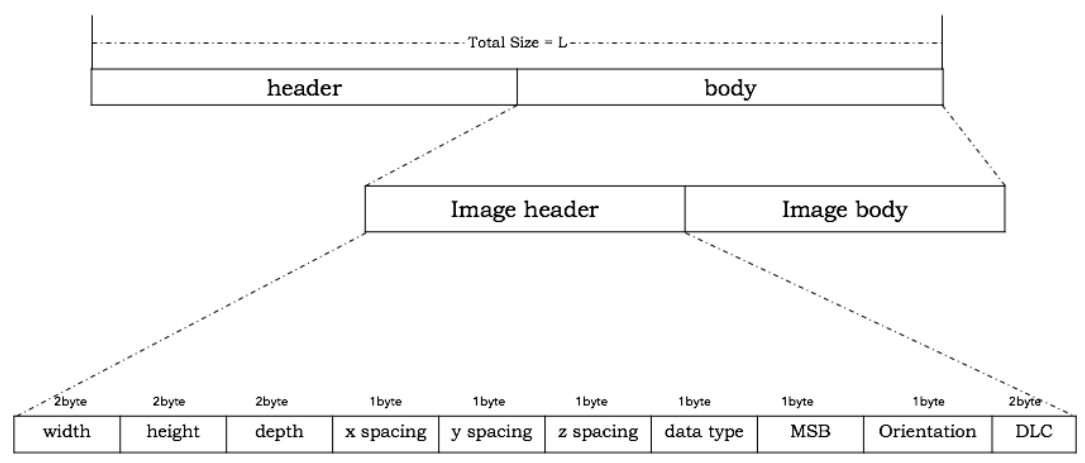

Figure 3. Composition of image data packets

As the medical image is relatively large, it will be divided into multiple messages and sent, as shown in Figure 4 where $\mathrm{L}_{\text {image }}$ represents the size of the image data; $\mathrm{L}_{\text {message }}$ represents the size of each message; $\mathrm{L}_{\text {header }}$ represents the size of the message header and $\mathrm{L}_{\text {imageHeader }}$ represents the size of the data packet header. The medical image will be split into $\mathrm{n}$ parts. 


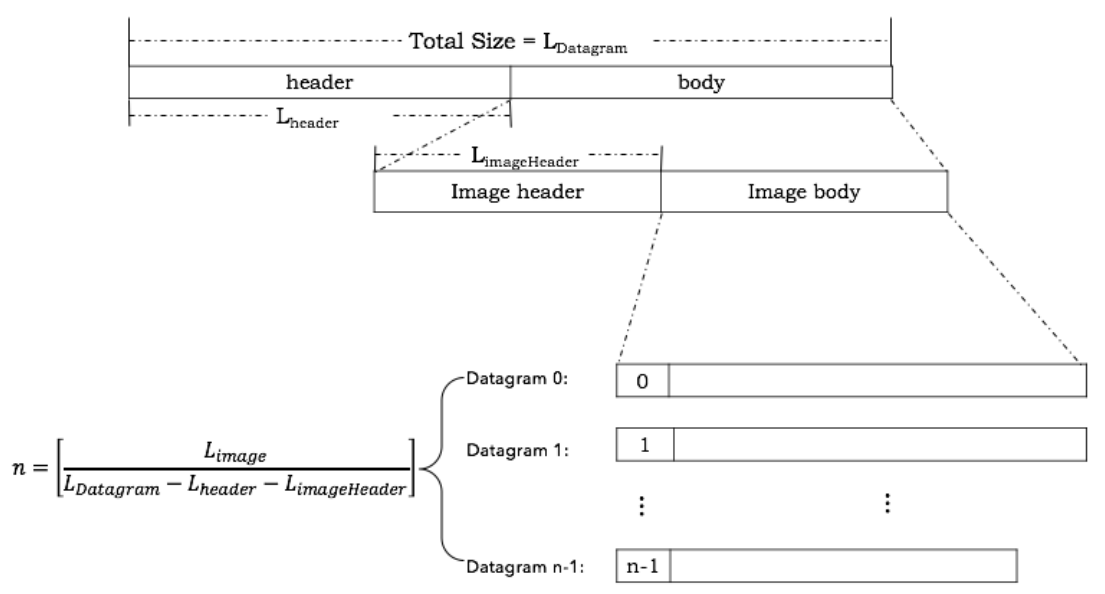

Figure 4. image data message content

\section{Network System Architecture}

In this paper, a set of network system framework that is suitable for multi-device communication is designed to meet the requirements of communication between multiple devices in vascular intervention robot system. This network system architecture, which can be used for variety of vascular intervention surgery robot system, can easily connect the various components of the system.

Details of Network System Architecture. A set of peer-to-peer TCP / IP communication is designed, as shown in Figure 5. This communication establishes two paths so that transmission and reception do not interfere with each other. In the process of communication with each other, there is no waiting problem between the incoming and outgoing message, which satisfies the requirement of real-time transmission of the message in the vascular intervention robot system. In the process of communication, through the transmission of messages, the session will be diagnosed to judge if the session is disconnected. Due to the establishment of a communication diagnostic mechanism, when the communication is interrupted, the session can be reconnected in time.

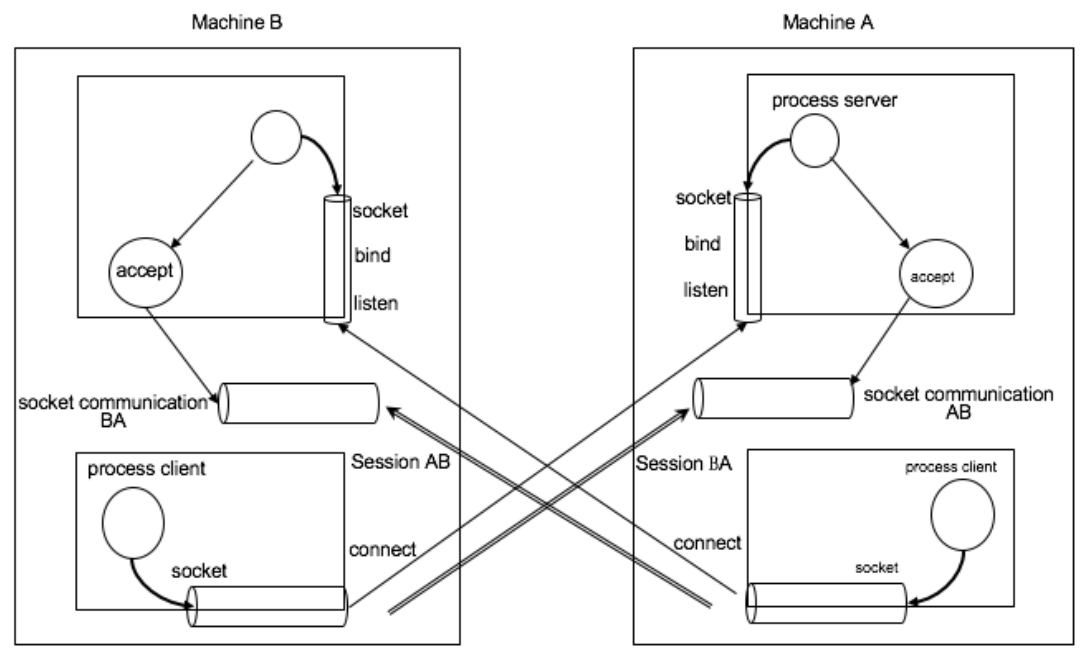

Figure 5. communication in vascular intervention surgery robot system

In this paper designed a set of vascular intervention surgery robot system communication architecture, based on the dual-channel TCP / IP communication method, so that this communication architecture can ensure the communication 
fluency and real-time capability, as shown in Figure 6. In each device, a server has been in the state of monitoring whether there is the active connection. Once receiving a signal that represents that some other devices actively try to connect to itself, it accepts the active connection and saves the socket and can manage the connection to the device. When the session between the devices is disconnected, the session can be reconnected because the connection to the device can be managed. The communication mode used in the communication architecture is asynchronous communication, which can ensure that So that there will be no communication jam. This communication architecture can be applied to a variety of vascular intervention surgery robot system to meet its real-time operation and feedback that communication needs.

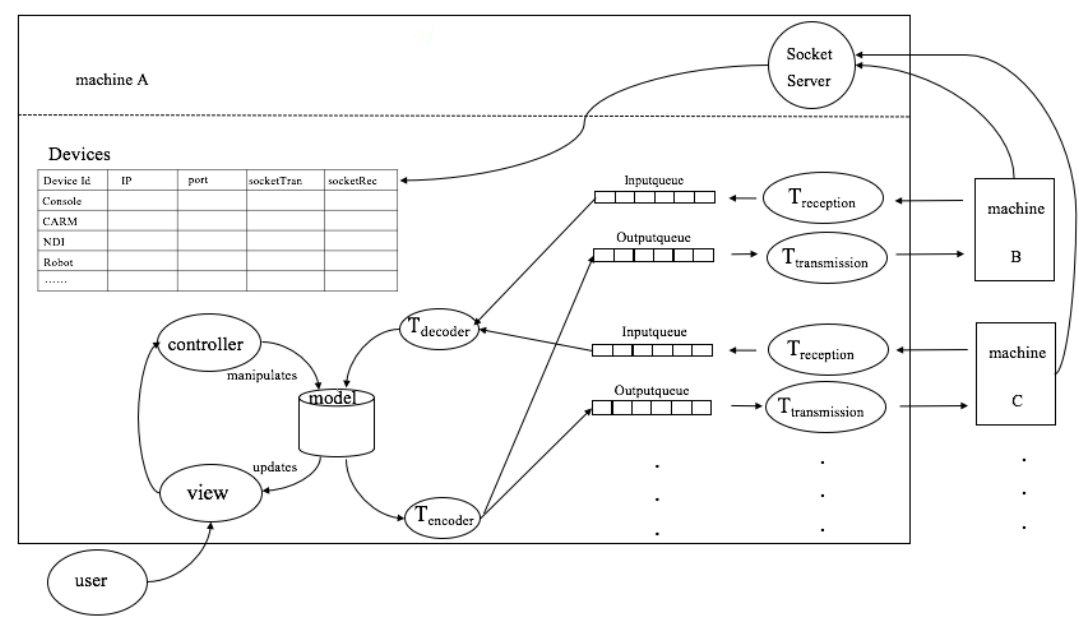

Figure 6. network communication architecture

\section{Application Instance and Evaluation of the Communication System}

To verify and ensure the reliability and rationality of the communication protocol and architecture design, the image-guided vascular interventional surgery(VIS) robot system uses the communication system (Fig. 7). The customized robot system is a remote-control robot, which must rely on the network communication system. The characteristic of vascular intervention requires the real-time capability and safety of the network communication system, so the evaluation experiment is necessary.

\section{Evaluation Experiment of the Communication System}

The customized VIS robot system aims to release the physicians from the ward and free from the X-ray radiation damage. Through thorough analysis of the whole procedure of the VIS, the interactive manipulation in the period of the operation mainly focuses on the advancement and rotation of the catheter. For the operation requirements, the slave manipulator is designed to operate the operation instrument. The slave manipulator is composed of four modules: advancement module, rotation module, force feedback module and support module. The advancement and rotation modules mainly achieve the function of the catheter/guidewire's advancement and rotation. The force feedback module obtains the forces and torques during the robotic operation, and the system imposes the feedback to the Omega master manipulator. Thus, the operator can sense the forces and torques, so that he/she can estimate the situation of the catheter/guidewire in the blood vessel and do the corresponding operation. The support module aims to ensure the smooth process of the catheter/guidewire's advancement. 
The robot system is composed of the main console platform (including master manipulator), CT image-guided system, VIS slave manipulator and communication system. The console runs at the real-time image-guided workstation, which is the brain of the whole system and sends the operation instruction to other components. CT image-guided system provides the real-time image collection and display. During the operation procedure, the operation of the Omega master manipulator will be transformed to the operation instruction sent to the slave manipulator. The slave manipulator will reappear the operation of the master manipulator. The process needs the cooperation of the real-time communication system. Moreover, the force feedback signal will impose to the Omega. The physicians can combine the real-time image display with the force feedback, so that they can do more accurate and correct operation.
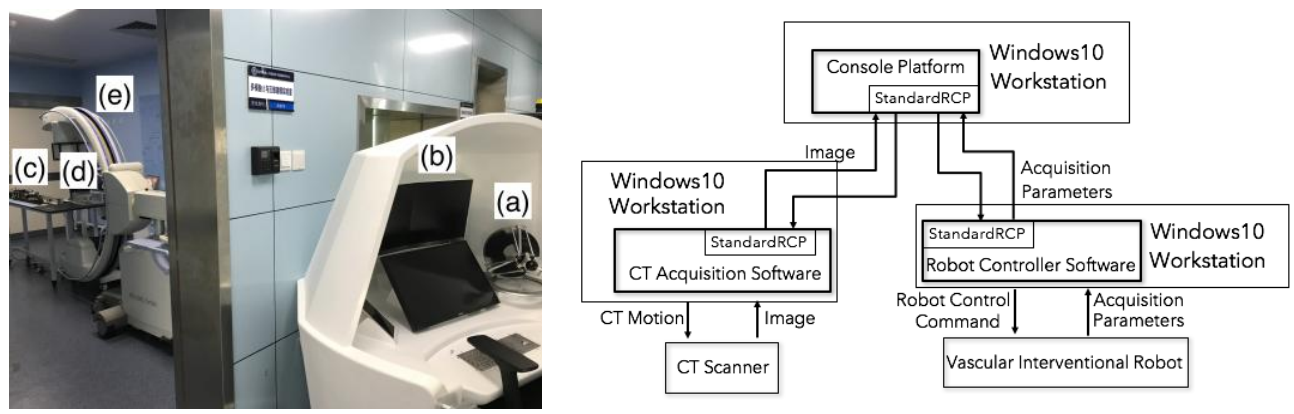

Figure 7. Vascular surgical robotic system, (a) Omega manipulator, (b) Main console platform, (c) Slave manipulator, (d) Vascular model, (e) CT Scanner

The vessel model experiment is conduct by the VIS robot system. To ensure the safety and reliability of the communication system, variety of message must be used to be transmitted. Meanwhile, the message owns interactive instruction and the time delay of the message transmission must be less than a certain threshold. The time delay of the force feedback data transmission and image data transmission is recorded. The time delay measures the time from start of the message coding generation from the sender to the end of the message decoding from the recipient. The design transmission frame rate of the force feedback data message is $2 \mathrm{n} f \mathrm{ps}$ ( $\mathrm{n}$ is from 1 to 10). The table 3 shows the time delay of different transmission frame rates of the force feedback data message. The table 4 shows the time delay of different sizes of the images data transmission in the condition of a set bandwidth. The tables indicate that the time delay of the message transmission keeps below a certain threshold. The time delay meets the requirement for the real-time capability of the communication system of the robot system.

Table 3 The latency of force feedback message in a variety of frame rate

\begin{tabular}{|c|c|c|c|}
\hline Frame rate (fps) & Mean (ms) & SD (ms) & Maximum (ms) \\
\hline 128 & 0.25 & 0.01 & 0.35 \\
\hline 256 & 0.31 & 0.03 & 0.45 \\
\hline 512 & 0.37 & 0.04 & 0.51 \\
\hline 1024 & 0.45 & 0.08 & 0.87 \\
\hline
\end{tabular}


Table 4 The latency for different size of image

\begin{tabular}{|c|c|c|c|}
\hline Size (KB) & Mean (ms) & SD (ms) & Maximum (ms) \\
\hline 128 & 3.42 & 0.02 & 3.45 \\
\hline 256 & 7.14 & 0.01 & 7.32 \\
\hline 512 & 15.22 & 0.03 & 18.24 \\
\hline 1024 & 30.45 & 0.03 & 38.56 \\
\hline 2048 & 64.34 & 0.03 & 80.78 \\
\hline
\end{tabular}

\section{Discussion}

In this paper, the communication protocol that is open, simple and extendable, satisfies the communication standard where many types of messages can be transmitted. For example, the latency of the sensor feedback message transmission and the image message are detected respectively in the application case of the communication protocol. During the operation, the accurate transmission of the sensor feedback message, which plays an important role in the robot control and the smooth operation of the operation, is very important. The surgeon can make judgments based on the feedback data to adjust the control of the robot to ensure the smooth operation. Through the transmission of image data, the surgeon can observe the image during the interventional surgery in real time, thereby performing image guidance during the operation and directly observing the position of the guide wire / catheter during the operation.

As we can see from Table 3, when the frame rate of sensor feedback message is less than $1024 \mathrm{fps}$, the latency is at the milliseconds level. As the number of channels increases and the frame rate increases, the CPU load increases. The frame rate of the sensor feedback message is typically less than $150 \mathrm{fps}$, so the sensor feedback data can be transmitted in real time, which is sufficient for most robotic-assisted vascular interventional surgery systems. As can be seen from Table 4, the transmission latency of image data compared to the sensor feedback data will be longer owing to the larger data, typically larger than $1 \mathrm{M}$. With the size of the image data increasing, the latency of image data will increase. The latency of image data becomes more important When multiple types of data are transmitted simultaneously through a single channel, because it may prevent the transmission of other types of data. Research on the transmission of multiple types of data at the same time shows that the image data should be divided into multiple messages to avoid blocking other data that needs to be transmitted more quickly. After the image data is divided into multiple messages, the latency of the image increases linearly with the increasing of the image data. But it is about 50 milliseconds basically according to the experiment, which can satisfy the requirement of the Real-time capability in intervention robot system.

The network communication protocol designed in this paper for robotic-assisted vascular interventional surgery is open, simple and extensible. In this paper, it proves to be able to process the data required with enough time and latency, according to the assessment to the efficiency of image data and sensor feedback data transmission. This protocol, which is more widely used, can be applied to all robotic-assisted vascular intervention systems and can facilitate the translation of research results into clinical and commercial applications.

\section{Conclusion}

The network communication protocol, which is open, simple and extensible, can satisfy the demand of the vascular interventional robotic system in this paper. In the 
application case of the communication protocol, the latency of the image data and the sensor feedback data are evaluated respectively. The latency of data transmission within the normal range meet requirements of the real-time capability. And this protocol is universal and applicable to all robotic-assisted vascular intervention systems, which can be applied in more scenes. The lack of protocol proposed in this paper is that it is only tested in the near-distance test, and the real-time performance of remote teleoperation is not evaluated and which will be improved in the future.

\section{Acknowledgement}

This research was financially supported by the following grants: National High Technology Research and Development Program of China (863 Program) (Nos. 2015AA043203), National Science Foundation Program of China (Nos. 61471349), Basic Discipline Layout Project of Shenzhen City (Nos. JCYJ20150731154850923), and Shenzhen Engineering Laboratory for Key Technologies on Intervention Diagnosis and Treatment Integration.

\section{References}

[1] Guo J, Guo S, Xiao N, et al. A new master-slave robotic catheter system[C]// Ieee/icme International Conference on Complex Medical Engineering. IEEE, 2011:610-613.

[2] Ma X, Guo S, Xiao N, et al. Development of a PID controller for a novel robotic catheter system[C]// Ieee/icme International Conference on Complex Medical Engineering. IEEE, 2011:64-68.

[3] Guo S. Analysis of robot-assisted endovascular interventional techniques [J]. Life science instruments In Chinese, 2013, ;(5):3-12. I

[4] Xia T, Baird C, Jallo G, et al. An integrated system for planning, navigation and robotic assistance for skull base surgery.[J]. International Journal of Medical Robotics + Computer Assisted Surgery Mrcas, 2008, 4(4):321.

[5] Kong X Z, Duan X G, Wang Y G. An integrated system for planning, navigation and robotic assistance for mandible reconstruction surgery[J]. Intelligent Service Robotics, 2016, 9(2):113-121.

[6] Riga C, Cheshire N. Future of robotics in vascular surgery[J]. Chinese medical journal, 2013, 126(3):550.

[7] Mura M, Parrini S, Ciuti G, et al. A computer-assisted robotic platform for vascular procedures exploiting 3D US-based tracking[J]. Computer Assisted Surgery, 2016, 21(1):63-79.

[8] Sotiris A, Christoforou E G, Panayides A S, et al. Medical telerobotic systems: current status and future trends:[J]. Biomedical Engineering Online, 2016, 15(1):96.

[9] Bzostek A, Kumar R, Hata N, et al. Distributed Modular Computer-Integrated Surgical Robotic Systems: Implementation Using Modular Software and Networked Systems[C]// International Conference on Medical Image Computing and ComputerAssisted Intervention. Springer-Verlag, 2000:969-978. 
[10] Tokuda J, Fischer G S, Papademetris X, et al. OpenIGTLink: an open network protocol for image-guided therapy environment[J]. International Journal of Medical Robotics \& Computer Assisted Surgery, 2009, 5(4):423. 